\title{
Avaliação dos hábitos de pacientes portadores de lesões cervicais não cariosas: um estudo observacional
}

\author{
Assessment of the habits of patients with non carious cervical injuries: an observational study \\ Evaluación de los hábitos de pacientes con lesiones cervicales no cariosas: un estudio observacional
}

Recebido: 04/03/2021 | Revisado: 10/03/2021 | Aceito: 11/03/2021 | Publicado: 19/03/2021

\author{
Bárbara Amurim Magalhães Pontes \\ ORCID: https://orcid.org/0000-0003-2508-7854 \\ Universidade de Fortaleza, Brasil \\ E-mail: barbaraamurim@hotmail.com \\ Gabriela Amorim Caldas \\ ORCID: https://orcid.org/0000-0001-7300-0368 \\ Universidade de Fortaleza, Brasil \\ E-mail: gabi.caldas01@hotmail.com \\ Karlos Eduardo Rodrigues Lima \\ ORCID: https://orcid.org/0000-0003-3127-9772 \\ Centro Universitário Católica de Quixadá, Brasil \\ E-mail: karlosed99@gmail.com \\ Talita Arrais Daniel Mendes \\ ORCID: https://orcid.org/0000-0003-3519-3618 \\ Universidade Federal do Ceará, Brasil \\ E-mail: talita_arrais@hotmail.com \\ Marcelo Victor Sidou Lemos \\ ORCID: https://orcid.org/0000-0002-2993-534X \\ Universidade de Fortaleza, Brasil \\ E-mail: marcelosidou@ hotmail.com
}

\begin{abstract}
Resumo
Objetivou-se avaliar os hábitos mais prevalentes em pacientes com lesões cervicais não cariosas, por meio de um estudo epidemiológico. Trata-se de um estudo epidemiológico de natureza transversal, no qual foram observados os hábitos deletérios de pacientes que possuem lesões cervicais não cariosas, por meio da aplicação de um questionário na clínica odontológica em uma universidade do Ceará. Tais participantes são brasileiros, com idade entre 18 a 60 anos de idade e apresentaram-se aptos a responder as perguntas presentes no questionário. Observou-se uma prevalência do gênero feminino na pesquisa. A maioria dos pacientes relatou o hábito de consumir alimentos ácidos e possuir hábitos parafuncionais. Fatores psicoemocionais e hábitos de higiene oral foram incluídos na pesquisa, como também a prevalência dos aspectos das lesões. Foi possível perceber que as lesões cervicais não cariosas denotam uma ampla variedade de características individuais e múltiplos fatores etiológicos que podem atuar na cavidade bucal. Por isso, faz-se necessário um estudo destas lesões para prevenir e controlar o seu processo de origem.
\end{abstract}

Palavras-chave: Abrasão dentária; Erosão dentária; Odontologia.

\begin{abstract}
The objective was to evaluate the most prevalent habits in patients with non-carious cervical lesions, through an epidemiological study. This is an epidemiological study of a cross-sectional nature, in which the deleterious habits of patients with non-carious cervical lesions were observed, through the application of a questionnaire in the dental clinic at a university in Ceará. Such participants are Brazilian, aged between 18 and 60 years old and were able to answer the questions in the questionnaire. There was a prevalence of the female gender in the research. Most patients reported the habit of consuming acidic foods and having parafunctional habits. Psycho-emotional factors and oral hygiene habits were included in the research, as well as the prevalence of aspects of injuries. It was possible to notice that noncarious cervical lesions denote a wide variety of individual characteristics and multiple etiological factors that can act in the oral cavity. Therefore, it is necessary to study these injuries to prevent and control their origin process.
\end{abstract}

Keywords: Dental abrasion; Dental erosion; Dentistry.

\section{Resumen}

El objetivo fue evaluar los hábitos más prevalentes en pacientes con lesiones cervicales no cariosas, mediante un estudio epidemiológico. Se trata de un estudio epidemiológico de carácter transversal, en el que se observaron los hábitos deletéreos de pacientes con lesiones cervicales no cariosas, mediante la aplicación de un cuestionario en la clínica dental de una universidad de Ceará. Dichos participantes son brasileños, tienen entre 18 y 60 años y pudieron 
responder las preguntas del cuestionario. Hubo una prevalencia del género femenino en la investigación. La mayoría de los pacientes refirieron el hábito de consumir alimentos ácidos y tener hábitos parafuncionales. Se incluyeron en la investigación factores psicoemocionales y hábitos de higiene bucal, así como la prevalencia de aspectos de las lesiones. Se pudo notar que las lesiones cervicales no cariosas denotan una amplia variedad de características individuales y múltiples factores etiológicos que pueden actuar en la cavidad bucal. Por tanto, es necesario estudiar estas lesiones para prevenir y controlar su proceso de origen.

Palabras clave: Abrasión dental; Erosión dental; Odontología.

\section{Introdução}

As lesões cervicais não cariosas (LCNC) caracterizam-se pela perda irreversível da estrutura dentária na região cervical, em nível de junção cemento-esmalte, sem envolvimento bacteriano. Estas apresentam etiologia multifatorial, sendo resultantes de um processo crônico. A sua presença na cavidade bucal pode ocasionar problemas estéticos, bucais e causar sensibilidade dentinária, tornando-se um grande problema de saúde pública (Souza \& Queiroz, 2019).

As LCNCs podem ocasionar a retenção de placa bacteriana, elevar a incidência de lesões cariosas, comprometer a integridade estrutural, vitalidade pulpar e também, pode levar ao aparecimento de hipersensibilidade dentinária devido à exposição dos túbulos dentinários causada pela perda de estrutura dentária. Esse episódio acomete com frequência pacientes com lesões cervicais não cariosas, apresentando dor aguda, provocada e de curta duração (Costa et al., 2018).

A etiologia das LCNCs é uma questão controversa que está em discussão. A etiologia multifatorial é amplamente aceita e tem como base a ação combinada de fatores que causam desgaste cervical nos dentes devido a: erosão ou biocorrosão (ácidos exógenos ou endógenos); abrasão (fatores relacionados à escovação); ou abfração, que pode ser ocasionada por fatores oclusais e hábitos parafuncionais, que aumentam o estresse na junção cemento-esmalte (Alvarez-Arenal et al., 2018).

A abrasão acontece devido a um atrito entre o dente e um agente exógeno, ocasionando um processo de desmineralização. A causa mais comum é a escovação traumática da estrutura dentária, além do uso do fio dental e palitos de dente (Dutra et al., 2016). Clinicamente, apresenta-se com um aspecto liso e brilhante, com forma em "V", frequentemente localizada na face vestibular, com margens bem definidas e a profundidade (Cruz da Silva et al., 2019).

A erosão dentária ou desgaste dentário erosivo pode ser considerado um problema de saúde bucal contemporâneo, causado por processos químicos sem envolvimento bacteriano. É ocasionada por ácidos quando o mesmo atinge a superfície dentária, podendo ser de origem intrínseca ou extrínseca (Maltarollo et al., 2020).

Para esses autores, os fatores intrínsecos da erosão que ocorrem na estrutura dentária estão relacionados com ácidos do sistema digestório que atingem a cavidade oral. Este evento ocorre devido a distúrbios gastrointestinais que podem causar vômito e refluxo. Já em relação aos fatores extrínsecos existentes, elas estão principalmente relacionadas à dieta ácida e também alguns medicamentos e drogas (Maltarollo et al., 2020).

As abfrações são lesões observadas principalmente nas superfícies vestibulares e são tipicamente em forma de cunha ou "V" e podem ser mais profundas que amplas, dependendo do estágio de progressão e dos fatores causais (Nascimento et al., 2016). A abfração é uma lesão na região cervical em forma de cunha afiada. Tem como principal fator etiológico o grande esforço oclusal excêntrico, tendo como consequência a flexão da estrutura dentária. A principal causa da abfração pode ser associada a uma sobrecarga oclusal (má oclusão) que, na maioria das vezes, ocasiona uma flexão do dente no limite amelocementário (Sousa et al., 2018).

Associando a mudança do estilo de vida e profissional da sociedade atual com o estresse gerado pelo dia a dia moderno, que eleva os níveis de pressão e ansiedade, é plausível acreditar que algumas pessoas estejam mais vulneráveis que outras e que o estado psicoemocional esteja vinculado ao aparecimento das lesões cervicais não cariosas, principalmente lesões de abfração. Pacientes que possuem doenças do sistema nervoso ou desequilíbrios emocionais prolongados adquirem mais facilmente hábitos parafuncionais e submetem mais os seus dentes a sobrecargas oclusais. Posto isto, diante de pacientes 
enquadrados neste quadro psicológico, deve-se suspeitar sempre da presença destas lesões (Molena et al., 2008).

Não somente na abfração, o estado psicoemocional do paciente deve ser levado em consideração. A bulimia nervosa é um transtorno da alimentação que possui, entre outras características, episódios recorrentes de compulsões periódicas, sendo a indução de vômito uma das principais características da doença. As compulsões apresentam-se associadas a estados de humor disfóricos, como depressão, situações negativas ou provocadoras de estresse, além de estar associada com o transtorno de ansiedade (Romaro \& Itokazu, 2002). Dessa forma, surge a ligação de doenças ligadas ao transtorno de ansiedade, como a bulimia nervosa, com a LCNC erosão, de forma que a indução de vômitos se caracteriza como fonte endógena de surgimento da lesão.

Existem estudos que mostram a ligação do transtorno de pânico (TP), caracterizado como um transtorno mental, com o refluxo gastroesofágico, que também se caracteriza como uma fonte endógena da erosão. O TP está também ligado a crises de ansiedade, visto que pode ser muitas vezes uma evolução destas (Duailibi et al., 2008).

Além dos fatores já apresentados, outro fator constantemente apresentado como etiologia das LCNC's são os fatores psicoemocionais, como ansiedade e depressão. Nesse contexto, a mudança do estilo de vida e profissional da sociedade atual com o estresse gerado pelo dia a dia moderno, que eleva os níveis de pressão e ansiedade, é plausível acreditar que algumas pessoas estejam mais vulneráveis que outras e que o estado psicoemocional esteja vinculado ao aparecimento das lesões cervicais não cariosas, principalmente lesões de abfração. Pacientes que possuem doenças do sistema nervoso ou desequilíbrios emocionais prolongados adquirem mais facilmente hábitos parafuncionais e submetem mais os seus dentes a sobrecargas oclusais, além de outras complicações como bulimia e refluxo gástrico (Romaro \& Itokazu, 2002). Posto isto, perante pacientes enquadrados neste quadro psicológico, deve-se suspeitar sempre da presença destas lesões (Molena et al., 2008).

Outro fator constantemente relacionado às LCNC's é a hipersensibilidade dentária, devido à perda continua de componentes, ocorre exposição de túbulos dentinários, resultando em uma maior susceptibilidade de respostas pulpares a estímulos externos como alterações osmóticas ou de temperatura (Costa et al., 2018).

Tendo em vista a grande prevalência de LCNC's e a grande variabilidade etiológica dos diferentes tipos de lesão, fazse necessário identificar os hábitos e condições bucais de pacientes portadores dessa doença para elaborar estratégias preventivas eficientes.

O objetivo do presente trabalho foi identificar os hábitos e condições orais de pacientes portadores de LCNC's que procuraram atendimento no curso de Odontologia da Universidade de Fortaleza.

\section{Metodologia}

Trata-se de um estudo observacional e transversal, de natureza qualitativa (Pereira et al., 2018), aprovado pelo comitê de ética e pesquisa com o número 3.293.866. O estudo foi executado por meio de aplicação de questionário no setor de triagem da clínica odontológica da Universidade de Fortaleza (UNIFOR), no período de janeiro a março de 2020.

Após exame inicial e verificação da presença de LCNC's, os participantes foram esclarecidos sobre os termos da pesquisa. A apresentação dos participantes ao setor de triagem ocorreu de forma voluntária. Todos os participantes assinaram o Termo de Consentimento Livre e Esclarecido. Nenhum diagnóstico referente aos níveis de ansiedade e depressão foi revelado aos participantes.

\section{Critérios de inclusão}

Pacientes que apresentaram pelo menos uma LCNC's, com nacionalidade brasileiro ou ter cidadania; estar incluso na faixa etária entre 18 e 60 anos de idade; apresentar-se apto a responder as perguntas presentes no questionário (capacidade cognitiva). 


\section{Critérios de exclusão}

Participantes que se encontravam em situação de vulnerabilidade social, tais como indivíduos sob reclusão penal, indígenas, menores de idade, pacientes com deficiências cognitivas diagnosticadas, dentre outros. Foram ainda excluídos participantes que durante a realização do exame clínico ou aplicação do questionário demonstraram algum desconforto ou optaram espontaneamente por serem removidos do grupo avaliado.

\section{Amostra}

Um total de 35 pacientes obedeceram aos critérios para inclusão na pesquisa, não sendo excluído nenhum paciente.

\section{Coleta de dados}

A aplicação do questionário foi realizada com auxílio de dois pesquisadores que passaram por uma calibração (Kappa $=0,9)$ para entendimento de como aplicar o questionário e como examinar as LCNCs.

A coleta foi realizada por meio da aplicação de um questionário de forma presencial. Os examinadores poderiam esclarecer possíveis dúvidas apresentadas pelos participantes, porém não poderiam induzir respostas, tendo em vista que as mesmas deveriam ser espontâneas. O desfecho primário do estudo foi a avaliação da ansiedade em pacientes que a apresentavam LCNC, aplicada por meio de uma escala hospitalar de ansiedade e depressão (Marcolino et al., 2007). Foi questionado aos pacientes também, como desfecho secundário idade, sexo, hábitos de higiene bucal, e hábitos dietéticos (Soares \& Grippo, 2017). Após realização do questionário foi realizado um exame clínico pelos pesquisadores calibrados, no qual foi necessário uma bandeja clínica, espelho intraoral número 05 , sonda exploradora, foco de iliminação, onde se podem observar as características das lesões apresentadas, assim como outras condições que acometiam a cavidade bucal.

Durante o exame não se realizou nenhum tipo de intervenção ou tratamento. Após participação na pesquisa, os voluntários foram encaminhados para tratamento nas diferentes disciplinas e estágios do curso de Odontologia da Universidade de Fortaleza, sem custos de tratamento.

\section{Análise de dados}

Todos os dados foram tabulados em planilha digital (Excel, Microsoft) e foram submetidos a uma análise estatística descritiva (SigmaPlot 14.0, SigmaStat). Os dados foram apresentados por meio de percentuais ou médias.

\section{Resultados}

Analisando os resultados obtidos, pode-se observar uma distribuição equilibrada entre voluntários do sexo masculino e feminino. A média de idade apresentada foi de 47,6 anos (Tabela 1). 
Tabela 1. Distribuição da amostra estudada quanto ao gênero e idade.

\begin{tabular}{lcc}
\hline SEXO & DISTRIBUIÇÃO & MÉDIA DE IDADE \\
\hline MASCULINO & $48,6 \%$ & $50,7(10,2)$ \\
FEMININO & $51,4 \%$ & $44,5(10,7)$ \\
TOTAL & $100 \%$ & $47,6(10,8)$ \\
\hline
\end{tabular}

Fonte: Autores (2021).

Quando avaliados os hábitos dietéticos, constatou-se que a maioria dos participantes fazia uso de refrigerantes, vinagre e frutas ácidas (Tabela 2). Destaca-se o fato de que (42,8\%) dos participantes consomem frutas pelo menos duas vezes por semana.

Tabela 2. Hábitos dietéticos da amostra estudada.

\begin{tabular}{ccccc}
\hline $\begin{array}{c}\text { HÁBITOS } \\
\text { DIETÉTICOS }\end{array}$ & Nunca & 1x por semana & 2x por semana & 1x por dia \\
\hline Refrigerante & $37,1 \%$ & $40 \%$ & $22,9 \%$ & $0 \%$ \\
Vinagre & $40 \%$ & $37,1 \%$ & $14,3 \%$ & $8,6 \%$ \\
Frutas ácidas & $14,3 \%$ & $28,5 \%$ & $42,8 \%$ & $14,4 \%$ \\
Bebida Alcoólica & $54,2 \%$ & $34,2 \%$ & $11,6 \%$ & $0 \%$ \\
Energéticos & $97,1 \%$ & $2,9 \%$ & $0 \%$ & $0 \%$ \\
Bebidas & $88,6 \%$ & $5,7 \%$ & $5,7 \%$ & $0 \%$ \\
Isotônicas & & & & \\
\hline
\end{tabular}

Fonte: Autores (2021).

Quanto ao questionamento sobre os aspectos orofaciais (Tabela 3), nota-se que o hábito de apertamento dos dentes foi o mais prevalente $(54,3 \%)$, seguido do hábito de ranger os dentes e tensionamento muscular orofacial, ambos com (37,2\%).

Tabela 3. Condições orofaciais da amostra estudada.

\begin{tabular}{ccccc}
\hline CONDIÇÃO & Nunca & $\begin{array}{c}\text { De vez em } \\
\text { quando }\end{array}$ & $\begin{array}{c}\text { Boa parte do } \\
\text { tempo }\end{array}$ & $\begin{array}{c}\text { Maior parte do } \\
\text { tempo }\end{array}$ \\
\hline $\begin{array}{c}\text { Ranger os dentes } \\
\text { Apertar os } \\
\text { dentes }\end{array}$ & $62,8 \%$ & $37,2 \%$ & $0 \%$ & $0 \%$ \\
Tensão muscular & $62,8 \%$ & $54,3 \%$ & $0 \%$ & $0 \%$ \\
Morder Objetos & $77,2 \%$ & $37,2 \%$ & $0 \%$ & $0 \%$ \\
\hline $\begin{array}{c}\text { DOR } \\
\text { OROFACIAL }\end{array}$ & $68,6 \%$ & $22,8 \%$ & $0 \%$ & $0 \%$ \\
\hline
\end{tabular}

Fonte: Autores (2021). 
Em relação aos fatores psicoemocionais (Tabela 4), observou-se que $(51,4 \%)$ se autodeclaram como sendo portadores de sintomas de ansiedade de depressão, porém somente $(37,1 \%)$ apresentaram sintomas de ansiedade e (20\%) apresentaram sintomas relativos à depressão segundo a Escala Hospitalar de Ansiedade e Depressão (Marcolino et al., 2007).

Tabela 4. Observação das condições de Ansiedade e Depressão apresentada pela mostra, segundo autopercepção e Escala Hospitalar de Ansiedade de Depressão (HADS).

\begin{tabular}{lcc}
\hline Condição & SIM & NÃO \\
\hline AUTOPERCEPÇÃO & $51,4 \%$ & $48,6 \%$ \\
ANSIEDADE & $37,1 \%$ & $62,9 \%$ \\
(HADS) & & \\
DEPRESSÃO & $20 \%$ & \\
(HADS) & & \\
\hline
\end{tabular}

Fonte: Autores (2021).

Avaliando-se os resultados referentes aos hábitos de higiene oral e condições orais da amostra estudada (Tabela 5), pode-se notar que $(48,6 \%)$ dos entrevistados faziam de escovas com cerdas médias ou duras. 48,5\% relataram apresentar sintomatologia dolorosa quando consumiam alimentos frios ou quentes. Outro achado relevante foi a prevalência de $(34,3 \%)$ dos pacientes apresentando facetas de desgaste.

Tabela 5. Hábitos de higiene oral e condições orais da amostra estudada.

\begin{tabular}{|c|c|c|c|c|}
\hline & 1x ao dia & 2 vezes ao dia & 3 vezes ao dia & $\begin{array}{c}4 \text { ou mais vezes } \\
\text { ao dia }\end{array}$ \\
\hline \multicolumn{5}{|l|}{ FREQUÊECIA } \\
\hline DA & $8,5 \%$ & $25,7 \%$ & $45,7 \%$ & $20,1 \%$ \\
\hline ESCOVAÇÃO & MÁCIA & MÉ & \multicolumn{2}{|r|}{ DURA } \\
\hline \multirow{2}{*}{$\begin{array}{c}\text { Tipo de escova } \\
\text { dental }\end{array}$} & \multirow[t]{2}{*}{$51,4 \%$} & \multicolumn{2}{|c|}{$42,8 \%$} & $5,8 \%$ \\
\hline & & \multicolumn{2}{|l|}{ SIM } & NÃO \\
\hline \multicolumn{2}{|c|}{$\begin{array}{c}\text { HIPERSENSIBILIDADE } \\
\text { (FRIO/CALOR) }\end{array}$} & $48,5 \%$ & \multicolumn{2}{|r|}{$51,5 \%$} \\
\hline uso do fio & & $74,2 \%$ & \multicolumn{2}{|r|}{$25,8 \%$} \\
\hline \multicolumn{2}{|c|}{$\begin{array}{c}\text { Tratamento Ortodôntico } \\
\text { prévio }\end{array}$} & $20 \%$ & \multicolumn{2}{|r|}{$80 \%$} \\
\hline Faceta de d & & $34,3 \%$ & \multicolumn{2}{|r|}{$65,7 \%$} \\
\hline
\end{tabular}


Ao todo foram avaliados 148 dentes contendo lesões cervicais não cariosas, resultando em uma média de 4,2 dentes acometidos por paciente. Destes, $70,3 \%$ apresentavam característica côncava rasa; já $26,3 \%$ apresentavam característica de cunha e 3,4\% tinham característica mista, onde não se pode identificar o formato da lesão de forma exata, tendo em vista que apresentavam regiões em aspecto de cunha e regiões de aspecto côncavo raso.

Tabela 6. Formato das lesões apresentadas pelos pacientes. Fortaleza, 2020.

\begin{tabular}{c|ccc}
\hline $\begin{array}{c}\text { Forma da lesão } \\
\text { apresentada }\end{array}$ & Côncava & Cunha & Mista \\
\hline$\%$ & $70,3 \%$ & $26,3 \%$ & $3,4 \%$ \\
\hline
\end{tabular}

Fonte: Autores (2021).

\section{Discussão}

O decréscimo de estrutura dentária na região cervical é um fator significativo para a presença de complicações biomecânicas na estrutura dentária. Conforme Machado et al. (2018), o enfraquecimento do esmalte e dentina na cervical dos elementos dentários é resultado de uma ação conjunta de tensões e fatores corrosivos, ocasionando assim, as LCNCs dentárias.

No presente estudo, há uma prevalência de lesões cervicais não cariosas em indivíduos com elevada faixa etária, predominantemente aos 50 anos de idade (Tabela 1). De acordo com a pesquisa de Figueiredo et al. (2016), este fato está relacionado a um maior tempo de exposição aos fatores etiológicos para surgimento de tais condições. O gênero não está diretamente relacionado ao aparecimento de lesões, porém, houve uma prevalência do gênero feminino $(51,4 \%)$, indo de encontro a outros estudos na literatura que mostram uma predominância de pacientes do gênero masculino com tal condição (Ferreira et al., 2020).

A ansiedade e a depressão tem sido alvo de vários estudos, pois ocasionam sérios danos à estrutura dental. As pessoas que apresentam essa enfermidade podem mostrar a presença de lesões cervicais não cariosas. Como observado na tabela 4, $(51,4 \%)$ dos pacientes tem autopercepção de estarem constantemente submetidos a situações de estresse ou depressão. Isso se deve ao fato de possuírem alguns hábitos, como os hábitos parafuncionais e a presença de componentes ácidos na alimentação, dentre outros. Foi relatado por Ferreira et al., (2012) que o estresse, a ansiedade, complicações no sono e algumas medicações são fatores que podem aumentar a frequência e a intensidade dos hábitos. Destaca-se a importância de diagnosticar tal condição se faz necessária, pois sabe-se que tratamento de LCNC, não consiste apenas em tratar a lesão propriamente dita, mas sim a etiologia propriamente dita dessa condição. Igualmente ao que este estudo demonstra dados na literatura traz que pacientes que possuem LCNC em sua maioria demonstram ansiedade, porém tal condição pode não ser causador de LCNC, porém pode agravar de maneira significativa (Ferreira et al., 2020).

Com base no estudo, pode-se observar um elevado percentual de pessoas $(42,8 \%)$ que possuem o habito de consumir frutas ácidas. Os pacientes especificaram o tipo e período de uso, assim mostrando uma maior frequência de consumo. $\mathrm{O}$ mesmo foi analisado por Kanzow et al. (2016), os quais observaram que os padrões alimentares mudaram e há uma maior adesão desses alimentos no cotidiano, assim justificando o aparecimento de lesões por biocorrosão. Como destacado também por Pereira et al. (2014), mostra que o consumo desses alimentos, juntamente com outros fatores como bulimia, diabetes, vômitos involuntários ou voluntários e sensação de queimação devem contribuir para o surgimento dessas lesões não cariosas.

Quanto aos hábitos dietéticos da amostra estudada, estima-se que há também um alto consumo de refrigerantes (22,9\%). Segundo Morimoto et al. (2014), a mesma bebida possui um pH por volta de 2.3, sendo potencialmente erosiva. A 
região cervical do elemento dentário possui esmalte com espessura fina, sendo uma das áreas mais atingidas. Além disso, a saliva não atua de forma rápida e abundante nessa região, portanto, o efeito tampão demora a ocorrer, facilitando, dessa forma, a presença de lesões cervicais não cariosas com características erosivas.

Em relação às condições orofaciais, há uma grande prevalência de hábitos deletérios, uma vez que grande parte dos pacientes $(54,3 \%)$ possui o hábito de apertar os dentes, gerando, consequentemente, sequelas maiores na porção cervical dos elementos dentários. O estudo de Sousa et al. (2019) relaciona que a abfração ocasiona a flexão do dente no limite amelocementário e o surgimento da lesão, sendo necessário, na maioria das vezes, o tratamento por estética, hipersensibilidade ou impactação alimentar.

Outro fator comumente encontrado na pesquisa está relacionado à dor orofacial $(68,6 \%)$, resultado de hábitos parafuncionais e fatores intrínsecos. Na pesquisa de Pirovani et al. (2019) consta que, além de promover destruição no periodonto, as forças excessivas podem provocar lesões no órgão dentário, no caso, as LCNC. Além disso, encontrou-se também na pesquisa o hábito de ranger os dentes e tensionamento muscular orofacial, ambos com (37,2\%). Vale salientar que no estudo de Pirovani et al. (2017), o bruxismo tem sido considerado um fator de risco para as DTM, bem como para as desordens musculares, ocasionando, muitas vezes, desconforto ao paciente.

Os hábitos de higiene oral são fundamentais para o equilíbrio da cavidade bucal; dessa forma, grande parte dos participantes da pesquisa $(45,7 \%)$, relataram que possuem o hábito de higienizar três vezes ao dia os elementos dentários com cerdas macias $(51,4 \%)$. Vale ressaltar que, na pesquisa realizada, foi observado também um grande número de pacientes $(42,8 \%)$ que usam escovas com cerdas médias. Conforme Barbosa et al. (2009), a técnica, força aplicada, frequência de escovação; rigidez das cerdas da escova dental podem levar ao aparecimento de lesões cervicais não cariosas, nomeada de abrasão.

Pode-se analisar que grande parte dos pacientes não possui hipersensibilidade dentinária $(51,5 \%)$, porém há um número significativo de pacientes que possuem (48,5\%). Segundo Cruz da Silva (2019), esta reação dolorosa está diretamente relacionada à exposição da dentina a estímulos químicos, táteis, térmicos ou osmóticos proveniente da cavidade bucal. Entretanto, a teoria mais aceita é a da hidrodinâmica dos fluídos dentinários.

Os estudos mostraram que existem três tipos de formatos de lesões cervicais não cariosas. Os formatos das lesões observadas nos pacientes da pesquisa, na tabela 6 , foram predominantemente lesões côncavas rasas $(70,3 \%)$, seguidas de lesões em formato de cunha (26,3\%) e, por fim, lesões mistas (3,4\%). Segundo Castro (2014); Carvalho (2010), as lesões, no exame clínico, caracterizadas como lesões côncavas, possuem como características diminuição do brilho dos dentes, apresentando uma superfície altamente polida e com grande lisura, formato côncavo, rasa e ângulos que não são nítidos, e em alguns casos mudança de cor.

Diante dos achados, sugere-se que novos estudos sejam realizados para observar os hábitos e condições bucais de pacientes portadores das LCNCs, a fim de se elaborar estratégias preventivas. A presença de LCNC não resulta apenas em alteração estética, mas também funcional com possibilidade de perda da eficiência mastigatória.

\section{Conclusão}

Foi possível perceber que as LCNCs denotam uma ampla variedade de características individuais e múltiplos fatores etiológicos que podem atuar na cavidade bucal. Visto que os mesmos dispõem intensidade, duração e frequência variáveis, podendo estar presentes em ampla faixa etária.

Portanto, faz-se necessário um estudo das lesões tão acometidas, para prevenir e controlar o processo de origem e para que seja possível observar o tipo de LCNC com essa correlação, além de definir a morfologia mais prevalente dessas LCNCs com pacientes que apresentam estresse e ansiedade. 


\section{Referências}

Alvarez-Arenal, A., Alvarez-Menendez, L., Gonzalez-Gonzalez, I., Alvarez-Riesgo, J. A., Brizuela-Velasco, A. \& deLlanos-Lanchares, H. (2019). Non-carious cervical lesions and risk factors: A case-control study. Journal of oral rehabilitation. 46(1), 65-75.

Barbosa, L. P. B., Prado-Jr, R. R. \& Mendes, R. F. (2009). Lesões cervicais não-cariosas: etiologia e opções de tratamento restaurador non-carious cervical lesions: origin and treatment options. Revista Dentística Online. 8(18), 5-10.

Carvalho, P. A. S. M. (2010). Lesões não cariosas: etiologia, plano de tratamentos e relações com profissões de estresse. Faculdade de Medicina Dentária da Universidade do Porto, Porto.

Castro, A. M. (2014). Lesões cervicais não cariosas: etiologia, opções de tratamento e durabilidade a longo prazo. Trabalho de Conclusão de Curso (Especialização em Dentística) - Faculdades Integradas do Norte de Minas, Brasília.

Costa, L. S., Alves, S. S. S., Lima, D. D. C., Dietrich, L., Santos-Filho, P. C. F. \& Martins, V. D. (2018). Lesão cervical não cariosa e hipersensibilidade dentinária: relato de caso clínico. Revista Odontológica do Brasil Central. 27(83), 247-251.

Duailibi, K., Lourenço, R. B., Mello, E. P., Magalhães, M. G., Netto, G. T. M., Diaz, M. C. N. \& Tannous, T. S. (2008). Refluxo gastroesofágico participando da cascata cognitiva do pânico. Jornal Brasileiro de Psiquiatria. 57(4), 280-282.

Dutra, L. C., Seabra, E. J. G., Silva, A. P., Martins, Y. V. M. \& Dutra, G. R. S. F. (2016). Lesões dentárias oriundas da oclusão. Revista da Universidade Vale do Rio Verde. 14(1), 44-52.

Ferreira, J. T. L., Lima, M. R. F. \& Pizzolato, L. Z. (2012). Relation between Angle Class II malocclusion and deleterious oral habits. Dental Press Journal of Orthodontics. 17(6), 111-117.

Ferreira, P. R. C., Queiroz, E. C., Santana, G. S., Lima, K. E. R., Fante, A. M., Lemos, M. V. S. \& Mendes, T. A. D. (2020). Association between anxiety and the presence of non-carious dental cervical lesions. Revista Brasileira de Odontologia. 77, 1863.

Figueiredo, V. M. G., Santos, R. L. \& Batista, A. U. D. (2016). Estudo das características e da hipersensibilidade de lesões cervicais não cariosas em pacientes com alterações oclusais. Revista da Faculdade de Odontologia-UPF. 21(3), 111-117.

Kanzow, P., Wegehaupt, F. J., Attin, T. \& Wiegand, A. (2016). Etiology and pathogenesis of dental erosion. Quintessence international. 47(4), $275-278$.

Machado, A. C., Neto, A. J. F., Junior, C. D. S., Vilela, A. L. R., Menezes, M. S., Teixeira, D. N. R., Cardoso, I. O. \& Soares, P. V. (2018). Influência do desequilíbrio oclusal na origem de lesão cervical não cariosa e recessão gengival: análise por elementos finitos. Revista Odontológica do Brasil Central. 27(83), 204-210.

Maltarollo, T. H., Pedron, I. G., Medeiros, J. M., Kubo, H., Martins, J. L. \& Shitsuka, C. (2020). The dental erosionis a problem! Research, Society and Development. 9(3), 1-10.

Marcolino, J. A. M., Mathias, L. A. S. T., Filho, L. P., Guaratini, A. A., Suzuki, F. M. \& Alli, L. A. C. (2007). Hospital Anxiety and Depression Scale: Study of Criterion Validity and Reliability with Preoperative Patients. Revista Brasileira de Anestesiologia. 57(1), 52-62.

Moelna, C. C. L., Rapoport, A., Rezende, C. P., Queiroz, C. M. \& Denardin, O. V. P. (2008). Relação entre lesões cervicais não cariosas e hábitos. Revista Brasileira Cirurgia Cabeça Pescoço. 37(5), 206-211.

Morimoto, S., Sesma, N., Agra, C. M., Guedes-Pinto, A. C. \& Hojo, K. Y. (2014). Erosão dental: etiologia, mecanismos e implicações. Journal of Biodentistry and Biomaterials. 4(1), 6-23.

Nascimento, M. M., Dilbone, D. A., Pereira, P. N. R., Duarte, W. R., Geraldeli, S. \& Delgado, A. J. (2016). Abfraction lesions: etiology, diagnosis, and treatment options. Clinical, cosmetic and investigational dentistry. 8, 79-87.

Pavani, A. P. S., Manfredi, G. G. P., Valle, L. A., Stuani, V. T., Michel, R. C., Damante, C. A., Zangrando, M. S. R. \& Sant’Ana, A. C. P. (2019). Relação entre trauma oclusal nas doenças periodontais e lesões cervicais não cariosas. Revista Uningá. 56(S5), 98-108.

Pereira, J. C., Netto, C. A. \& Gonçalves, S. A. (2014). Dentística: uma abordagem multidisciplinar. Artes Médicas Editora.

Pereira, A. S., Shitsuka, D. M., Parreira, F. J. \& Shitsuka, R. (2018). Metodologia da pesquisa científica. UFSM.

Pirovanai, B. O. (2017). Correlação entre bruxismo, qualidade de vida e dor em portadores de desordens temporomandibulares. Trabalho de Conclusão de Curso (Odontologia) - Universidade Estadual Paulista, Araçatuba.

Romaro, R. A. \& Itokazu, F. M. (2002). Bulimia nervosa: revisão da literatura. Psicologia: Reflexão e Crítica. 15(2), 407-412.

Cruz da Silva, E. T., Gadelha Vasconvelos, R., Gadelha Vasconcelos, M. (2019). Lesões cervicais não cariosas: considerações etiológicas, clínicas e terapêuticas. Revista Cubana de Estomatologia. 56(4), 1-15.

Soares, P. V. \& Grippo, J. O. (2017). Non-Carious Cervical Lesions and Cervical Dentin Hypersensitivity: Etiology, diagnosis and treatment. Quintessence. Portuguese.

Sousa, L. X., Cruz, J. H. A., Melo, W. O. S., Freire, S. C. P., Ribeiro, E. D. \& Freire, J. C. P. (2018). Abfração dentária: um enfoque sobre a etiologia e o tratamento restaurador. Archives of Health Investigation. 7(2), 51-53.

Souza, J. W. C. \& Queiroz, M. C. (2019). Conhecimentos clínicos sobre diagnóstico de lesões cervicais não cariosas: revisão bibliográfica. Trabalho de Conclusão de Curso (Odontologia) - Universidade Estadual da Paraíba. 